\title{
ESTUDIO DE LOS ESPACIOS PROFESIONALES ACTUALES DE LA PEDAGOGÍA: LA VOZ DEL ALUMNADO Y DE LOS PROFESIONALES
}

\author{
Study of the Current Professional Spaces of the Pedagogy: \\ The Opinion of the Students and the Professionals
}

Jon ALTUNA URDIN, Esther CRUZ IGLESIAS, Ana AIERBE BARANDIARAN, Alba MADINABEITIA EZKURRA e Inazio MARKO JUANIKORENA

Universidad del País Vasco.España.

jon.altuna@ebu.eus; esther.cruz@ebu.eus; ana.aierbe@ebu.eus;

alba.madinabeitia@ebu.eus; inazio.marko@ebu.eus

https://orcid.org/0000-0002-1847-8083; https://orcid.org/0000-0001-6093-9309; https://orcid.org/0000-0003-3933-0587; https://orcid.org/0000-0002-8907-4764;

bttps://orcid.org/0000-0001-7005-1960

Fecha de recepción:27/05/2020

Fecha de aceptación: 23/11/2020

Fecha de publicación en línea: 01/03/2021

Cómo citar este artículo: Altuna Urdin, J., Cruz Iglesias, E., Aierbe Barandiaran, A., Madinabeitia Ezkurra, A. y Marko Juanikorena, I. (2021). Estudio de los espacios profesionales actuales de la Pedagogía: la voz del alumnado y de los profesionales. Teoría de la Educación. Revista Interuniversitaria, 33(2), 195-215. https://doi.org/10.14201/ teri. 23714

RESUMEN

Introducción: Este artículo se enmarca en una investigación que se ha realizado en el grado de Pedagogía de la Facultad de Educación, Filosofía y Antropología de la UPV/EHU. Objetivo: Identificar las necesidades formativas del alumnado con relación a las competencias transversales de la titulación de cara a la inserción laboral del 
mismo. Método: El diseño utilizado es exploratorio, descriptivo y longitudinal. Se parte del análisis de la voz del alumnado, realizando un cuestionario y unos focus group referidos a las salidas profesionales y las competencias transversales. Posteriormente, se recoge la voz de los profesionales de la Pedagogía respecto a las dos cuestiones anteriores mediante un cuestionario y entrevistas individuales. Resultados: El alumnado piensa que la salida profesional de teoría pedagógica, evaluación e investigación es la que más se trabaja en el grado. Detectan necesidades formativas relacionadas con orientación laboral, el uso de la TICs y la falta de interacción entre el mundo académico y profesional. Respecto a las competencias transversales, el alumnado piensa que se trabajan de forma implícita y no explícita y apuntan que las competencias de creatividad e innovación y aprender a aprender son poco trabajadas. Los profesionales valoran mucho todas las competencias transversales, pero son las de aprender a aprender y comunicación las que obtienen puntuaciones más altas. Discusión: Si los profesionales piensan que las competencias de aprender a aprender y comunicación son las más valoradas y el alumnado piensa que no se trabajan lo suficiente y que no se hace de manera explícita, el profesorado debería diseñar un proceso de mejora del grado en esta línea. Conclusiones: De cara a la mejora del grado de Pedagogía, hay que emprender acciones para explicitar el trabajo de las competencias transversales e incorporar de forma explícita otras como la adaptación al cambio y la capacidad para atraer la atención.

Palabras clave: Pedagogía; enseñanza superior; desarrollo educativo; cualificación profesional; calidad de la educación.

\section{ABSTRACT}

Introduction: This article is part of an investigation that has been carried out in the degree of pedagogy of the Faculty of Education, Philosophy, and Anthropology of the UPV/EHU. Objective: To identify the training needs of the students in relation to the transversal competences of the degree with a view to their insertion in the labour market. Method: The design used is exploratory, descriptive, and longitudinal. It is based on the analysis of the voice of the students, carrying out a questionnaire, and some focus groups regarding the professional opportunities and the transversal competences. Subsequently, the voice of the teaching professionals is collected with respect to the two previous questions by means of a questionnaire and individual interviews. Results: The students think that the professional output of pedagogical theory, evaluation, and research is the one that is most worked on in the degree. They detect training needs related to work orientation, the use of ICTs, and the lack of interaction between the academic and professional worlds. With regard to the transversal skills, students think that they are worked on implicitly and not explicitly and point out that the skills of creativity and innovation and learning to learn are little worked on. Professionals highly value all transversal competences, but it is learning to learn and communication that obtains the highest scores. Discussion: If professionals think that the learning to learn and communication skills are the most valued and students think that they are not worked on much and that it is not done in an explicit way, 
JON ALTUNA, ESTHER CRUZ, ANA AIERBE, ALBA MADINABEITIA E INAZIO MARKO ESTUDIO DE LOS ESPACIOS PROFESIONALES ACTUALES DE LA PEDAGOGÍA: LA VOZ DEL ALUMNADO Y DE LOS PROFESIONALES

teachers should design a process to improve the degree in this line. Conclusions: In order to improve the degree of pedagogy, we must undertake actions to make explicit the work of the cross-curricular skills and to explicitly incorporate others such as adaptation to change and the ability to attract attention.

Key words: Pedagogy; higher education; educational development; vocational qualification; educational quality.

\section{INTRODUCCIÓN}

Desde que se crearon las primeras facultades de Pedagogía hasta la fecha ha habido una gran evolución respecto al quehacer profesional del titulado en Pedagogía. En este artículo, nos vamos a situar en la actualidad más reciente y, partiendo del Real Decreto 1393/2007 donde se recoge la reordenación de las enseñanzas universitarias oficiales en el nuevo Espacio Europeo de Educación Superior EEES, se va a reflexionar en torno a esta temática.

En los últimos tiempos se han producido profundos cambios estructurales en los contextos en los que trabaja el profesional de la Pedagogía. Han desaparecido espacios profesionales que tradicionalmente habían estado ligados a la profesión y han aparecido otros nuevos, y, por tanto, se hace necesario recoger las necesidades sociales para adaptar la oferta de formación a esas realidades cambiantes.

Por otra parte, la tasa de empleabilidad de los profesionales de la Pedagogía es muy alta, pero la figura del pedagogo o pedagoga tiene escasa visibilización social porque las diferentes administraciones no reivindican, de manera preferente u obligatoria, esta figura profesional en determinados puestos de trabajo.

Para dar respuesta a esta realidad, en 2014 se comenzó una línea de investigación en la Facultad de Educación, Filosofía y Antropología del País Vasco (UPV/ EHU) que se ha prolongado hasta la actualidad. Así, se ha analizado, por una parte, la opinión del alumnado de Pedagogía sobre la adquisición de competencias transversales claves para su futuro profesional de cara a la mejora del grado y, por otra, se ha recogido la percepción de los profesionales de la Pedagogía sobre las competencias transversales necesarias para el desempeño de la profesión en general y sobre sus ámbitos laborales en particular. La confluencia de estas dos percepciones unidas a la reflexión del profesorado del grado, sientan las bases del análisis de las competencias necesarias, tanto específicas como transversales, para preparar mejor a los futuros profesionales de la Pedagogía.

\subsection{La voz del alumnado y de los profesionales para la mejora continua}

La calidad educativa que brindan las universidades es un factor de progreso de la sociedad y la percepción del alumnado, a través de las encuestas de satisfacción, es un indicador para evaluarla. Si a éstos incorporamos la percepción de los 
JON ALTUNA, ESTHER CRUZ, ANA AIERBE, ALBA MADINABEITIA E INAZIO MARKO ESTUDIO DE LOS ESPACIOS PROFESIONALES ACTUALES DE LA PEDAGOGÍA: LA VOZ DEL ALUMNADO Y DE LOS PROFESIONALES

profesionales que trabajan en el área y lo contrastamos con lo que se ofrece desde las titulaciones, la información adquiere otra dimensión que ayuda a la mejora continua de los propios grados.

Algunos autores afirman que en los procesos de mejora de los planes de estudio se debería tener en cuenta la voz del alumnado (Rodríguez- Martín, 2017; Salinas, Morales y Martínez, 2008; Calvo y Susinos, 2010) y piensan que es necesario que los y las estudiantes expresen sus necesidades e intereses sobre su paso por el grado.

Diversos autores señalan que la calidad de un servicio educativo se mide por la satisfacción del receptor de dicho servicio, en este caso, la calidad educativa se mediría por la satisfacción del alumnado (Surdez, Sandoval y Lamoyi, 2018).

Es necesario tener en cuenta que no siempre que se da una evaluación se produce una mejora, es necesario dilucidar una serie de indicadores en el proceso de evaluación que nos ayuden a mejorar la calidad educativa (Calvo y Susinos, 2010). La investigación que nos ocupa parte de una evaluación que trata de encontrar indicadores para guiar el proceso de mejora del Grado de Pedagogía. Por todo ello, es hoy más necesario que nunca pensar en la universidad que queremos generando una comunidad científica que discuta la mejora de la docencia y del aprendizaje del alumnado desde un deseo de cambio que no silencie las experiencias del profesorado, ni la de las y los estudiantes, recuperando así, las voces de los protagonistas de la educación superior (Calvo y Susinos, 2010), sin olvidar, como punto de contraste fundamental, la experiencia de los profesionales que desarrollan su labor en el área profesional en la que queremos formar al alumnado.

\subsection{Competencias transversales de los profesionales de la Pedagogía}

Uno de los retos principales de las universidades de calidad, es la de formar profesionales capaces de adaptarse a un mundo cambiante, incierto y complejo, que exige de las personas en formación un proceso de transformación, necesario para vivir en la sociedad actual caracterizada por su estado permanente de cambio a nivel global (Hargreaves, 2003). Las distintas universidades, e incluso la formación profesional, a partir de la declaración de Bolonia en 1999 y de la Conferencia Mundial de Educación Superior de 2006, se han replanteado su papel con relación al conocimiento, a la sociedad y al mundo profesional para dar respuesta a dichas necesidades (Eizagirre, Altuna y Fernández, 2017).

Este proceso de replanteamiento del papel de la universidad en la sociedad implica especificar en el currículum, además de las competencias científico-técnicas, las competencias transversales que han de adquirir los y las estudiantes para afrontar los retos de un mundo globalizado en el que dominan las tecnologías, la sobreinformación y en el que también hay que enfrentarse a retos sociales tales como: el crecimiento económico, la sostenibilidad socio-ambiental y la prosperidad basada en la equidad social (Rekalde y Bujan, 2014). 

LA VOZ DEL ALUMNADO Y DE LOS PROFESIONALES

Centrándonos en el Grado de Pedagogía, a la hora de definir las competencias transversales partimos de diferentes documentos. Por una parte, las investigaciones realizadas entre los años 2016 y 2018 en la facultad de Educación, Filosofía y Antropología de la UPV/EHU en la que se definían las competencias transversales propias del grado de Pedagogía (Eizagirre, Altuna, Pikabea, Marko, y Pérez, 2017) y, por otra, en el catálogo de las Competencias transversales de la UPV/ EHU (Uranga Cruz, Gil, Losada y Ruiz de Gauna, 2019), en donde, basándose en el Informe de la III Conferencia de Educación Superior de la región Asia-Pacífico de la Unesco (2016) se plantean las Competencias Transversales propias de la esta Universidad. No se ha optado por la clasificación de las competencias en instrumentales, interpersonales y sistémicas planteadas en el Libro Blanco de la Pedagogía y la Educación Social (Villa, 2004) porque se utilizaron las 20 competencias resultantes de dicha clasificación para la definición de las competencias propias del Grado de Pedagogía.

Dentro de la investigación del 2016-2018 realizada en la facultad de Educación, Filosofía y Antropología se avanzó en la definición de las 5 competencias transversales propias del grado de Pedagogía (Marko, Pikabea, Altuna, Eizagirre y Perez-Sostoa, 2019). Estas competencias transversales son las siguientes: a) Aprender a aprender: la capacidad para proseguir y persistir en el aprendizaje, organizar el propio aprendizaje, lo que conlleva realizar un control eficaz del tiempo y la información, individual y grupalmente. Conlleva habilidades de aprendizaje como la autonomía, la toma de decisiones, la competencia digital, y la implicación. b) Comunicación oral: la entendemos como competencia comunicativa. Es la habilidad del que utiliza la lengua para negociar, intercambiar e interpretar significados con un modo de actuación adecuado. Supone la capacidad de expresar conceptos e ideas, de forma clara y comprensible, expresar la propia comprensión que ha desarrollado el alumnado sobre un tema, desarrollar habilidades de comunicación y presentación de ideas. c) Creatividad e Innovación: habilidad para la producción de ideas y para la génesis y resolución de los problemas de forma novedosa. Así mismo, supone saber poner en marcha dichas ideas nuevas y útiles, o integrar nuevos procesos, productos etc. dentro del sistema. d) Trabajo en equipo: es la capacidad de integrarse en un grupo, interdisciplinar o no, colaborando de forma activa, para lograr objetivos comunes, intercambiando informaciones, asumiendo responsabilidades, resolviendo dificultades y contribuyendo a la mejora y al desarrollo colectivo. e) Comunicación escrita: la capacidad de expresarse y comprender ideas, conceptos y sentimientos en su lengua o lenguas maternas con un adecuado nivel de uso. Supone la capacidad de expresar conceptos e ideas, de forma clara y comprensible, expresar la propia comprensión que ha desarrollado el alumnado sobre un tema, desarrollar habilidades de comunicación y presentación de ideas.

Estas competencias, a su vez, se dividieron en 5 subdimensiones o subcompetencias: 
TABLA 1

Competencias y subcompetencias de la titulación de Pedagogía de la Universidad del País Vasco

\begin{tabular}{|c|c|}
\hline Competencias & Subdimensiones o Subcompetencias \\
\hline \multirow{6}{*}{ Comunicación oral } & Capacidad para la exposición estructurada de la presentación \\
\hline & $\begin{array}{l}\text { Capacidad para la utilización adecuada de medios de apoyo a la } \\
\text { presentación (soportes, TIC...) }\end{array}$ \\
\hline & Lenguaje, voz y velocidad apropiada acorde al entorno \\
\hline & Capacidad de atracción de la atención \\
\hline & Lenguaje no verbal adecuado \\
\hline & $\begin{array}{l}\text { Conducción del mensaje y adecuación a la interacción con el re- } \\
\text { ceptor }\end{array}$ \\
\hline \multirow{5}{*}{ Comunicación escrita } & Capacidad para escribir de una manera correcta y clara \\
\hline & $\begin{array}{l}\text { Capacidad para la argumentación y justificación de ideas y opinio- } \\
\text { nes escritas. }\end{array}$ \\
\hline & $\begin{array}{l}\text { Capacidad para utilizar de forma precisa la terminología pedagó- } \\
\text { gica }\end{array}$ \\
\hline & Capacidad para la producción de diferentes tipos de textos \\
\hline & Capacidad para usar fuentes diversas \\
\hline \multirow{6}{*}{ Trabajo en equipo } & Capacidad para la planificación del equipo \\
\hline & Capacidad de coordinación-cooperación \\
\hline & Capacidad de comunicación (interna grupo-equipo) \\
\hline & Capacidad para el seguimiento y Feedback \\
\hline & $\begin{array}{l}\text { Capacidad para la resolución de conflictos y problemas colabora- } \\
\text { tivos }\end{array}$ \\
\hline & Capacidad de apertura, integración y ajustes del equipo \\
\hline \multirow{5}{*}{ Aprender a aprender } & $\begin{array}{l}\text { Capacidad para buscar, seleccionar, organizar, gestionar la informa- } \\
\text { ción con rigor científico-académico }\end{array}$ \\
\hline & Capacidad para la autonomía y autorregulación en el aprendizaje \\
\hline & $\begin{array}{l}\text { Competencia digital acorde a las exigencias educativas y profesio- } \\
\text { nales }\end{array}$ \\
\hline & $\begin{array}{l}\text { Capacidad para mantener una actitud positiva y de motivación ha- } \\
\text { cia el aprendizaje }\end{array}$ \\
\hline & Capacidad para la crítica y el compromiso ético y responsable \\
\hline
\end{tabular}


JON ALTUNA, ESTHER CRUZ, ANA AIERBE, ALBA MADINABEITIA E INAZIO MARKO ESTUDIO DE LOS ESPACIOS PROFESIONALES ACTUALES DE LA PEDAGOGÍA: LA VOZ DEL ALUMNADO Y DE LOS PROFESIONALES

\begin{tabular}{|l|l|}
\hline Competencias & Subdimensiones o Subcompetencias \\
\hline \multirow{5}{*}{$\begin{array}{l}\text { Creatividad e } \\
\text { innovación }\end{array}$} & $\begin{array}{l}\text { Capacidad para adaptarse a las situaciones cambiantes, modifican- } \\
\text { do la conducta para integrarse, con versatilidad y flexibilidad }\end{array}$ \\
\hline $\begin{array}{l}\text { Capacidad para proponer nuevas soluciones a problemas pedagó- } \\
\text { gicos convencionales }\end{array}$ \\
\cline { 2 - 2 } $\begin{array}{l}\text { Capacidad para generar nuevos problemas y/o cuestiones peda- } \\
\text { gógicas }\end{array}$ \\
\cline { 2 - 2 } $\begin{array}{l}\text { Capacidad para valorar, defender y promover con iniciativa ideas/ } \\
\text { propuestas creativas en términos de originalidad, utilidad y viabi- } \\
\text { lidad }\end{array}$ \\
\cline { 2 - 2 } \\
$\begin{array}{l}\text { Orientación a la calidad, utilizando indicadores de evaluación, y } \\
\text { buscando la mejora continua }\end{array}$ \\
\cline { 2 - 2 } & Capacidad de apertura, integración y ajustes del equipo \\
\hline
\end{tabular}

Fuente: Marko et al. (2019)

Cuando se analizan las subcategorías que se plantearon dentro de las competencias elaboradas para el Grado de Pedagogía y se comparan con las Competencias Transversales propuestas en el Catálogo de la UPV/EHU (Uranga et al., 2019), se percibe que todas las competencias transversales de la UPV/EHU se tienen en cuenta para la definición de las propias del Grado. Por lo tanto, en esta investigación se decide partir de las competencias propias para el Grado de Pedagogía definidas por Marko et al. (2019).

\subsection{Salidas profesionales de la Pedagogía}

En la universidad actual, la empleabilidad del alumnado es un indicador del éxito de la formación recibida. Ya en la cumbre de Lisboa del año 2000 se muestra clara la postura del Consejo Europeo al establecer la promoción de la empleabilidad como requisito para la construcción de una sociedad basada en el conocimiento, competitiva y dinámica. Son las competencias transversales las que más se adecúan al discurso del EEES (Prades, 2006) y las que integran los elementos necesarios de los mundos profesional y académico, equipando a los futuros graduados de estrategias para adaptarse a las nuevas necesidades del mercado laboral (Eizagirre y Altuna, 2014).

Para el estudio de las salidas profesionales del Grado de Pedagogía se han analizado, por una parte, en el libro Blanco para la Pedagogía y la educación social (Villa, 2004), en el que se recogen los ámbitos profesionales donde el profesional de la Pedagogía tiene cabida, y por otro, en un estudio realizado en la Facultad de Educación Filosofía y Antropología de la UPV/EHU que analiza el ámbito profesional donde se sitúa el alumnado egresado de la propia Facultad (Eizagirre et al., 2017). Desde el planteamiento de la Aneca, en la Pedagogía se definen seis ámbitos, y cada uno de ellos está compuesto por una serie de perfiles profesionales: Administración pública, donde se incluyen los perfiles de gestor de centros educativos, inspector y supervisor de la administración educativa y evaluador de sistemas e instituciones 
educativas; Orientación psicopedagógica en el que están los perfiles de orientador personal, académico y profesional y orientador familiar; Desarrollo y evaluación de procesos y medios didácticos en el que se encuentran diseñador de recursos, diseñador y evaluador de procesos de enseñanza-aprendizaje y materiales curriculares y formador pedagógico de la función docente; Formación en organizaciones laborales con las figuras del consultor y gestor de formación en las organizaciones y formador de formadores; Formación e inserción de personas adultas con los perfiles de orientador socio-laboral y formador de personas adultas; Y, por último, atención socioeducativa a la diversidad con el pedagogo especialista en atención a la diversidad y el dinamizador para la inserción sociolaboral.

En el estudio de la Facultad de Educación Filosofía y Antropología de la UPV/ EHU, no solo se definen las áreas o ámbitos profesionales sino que se elabora un mapa de salidas profesionales del pedagogo/a, quedando configurado con 3 ámbitos de trabajo diferenciados y con 5 espacios profesionales en cada uno de esos ámbitos:

\section{IMAGEN 1}

Mapa de los ámbitos profesionales de la Pedagogía

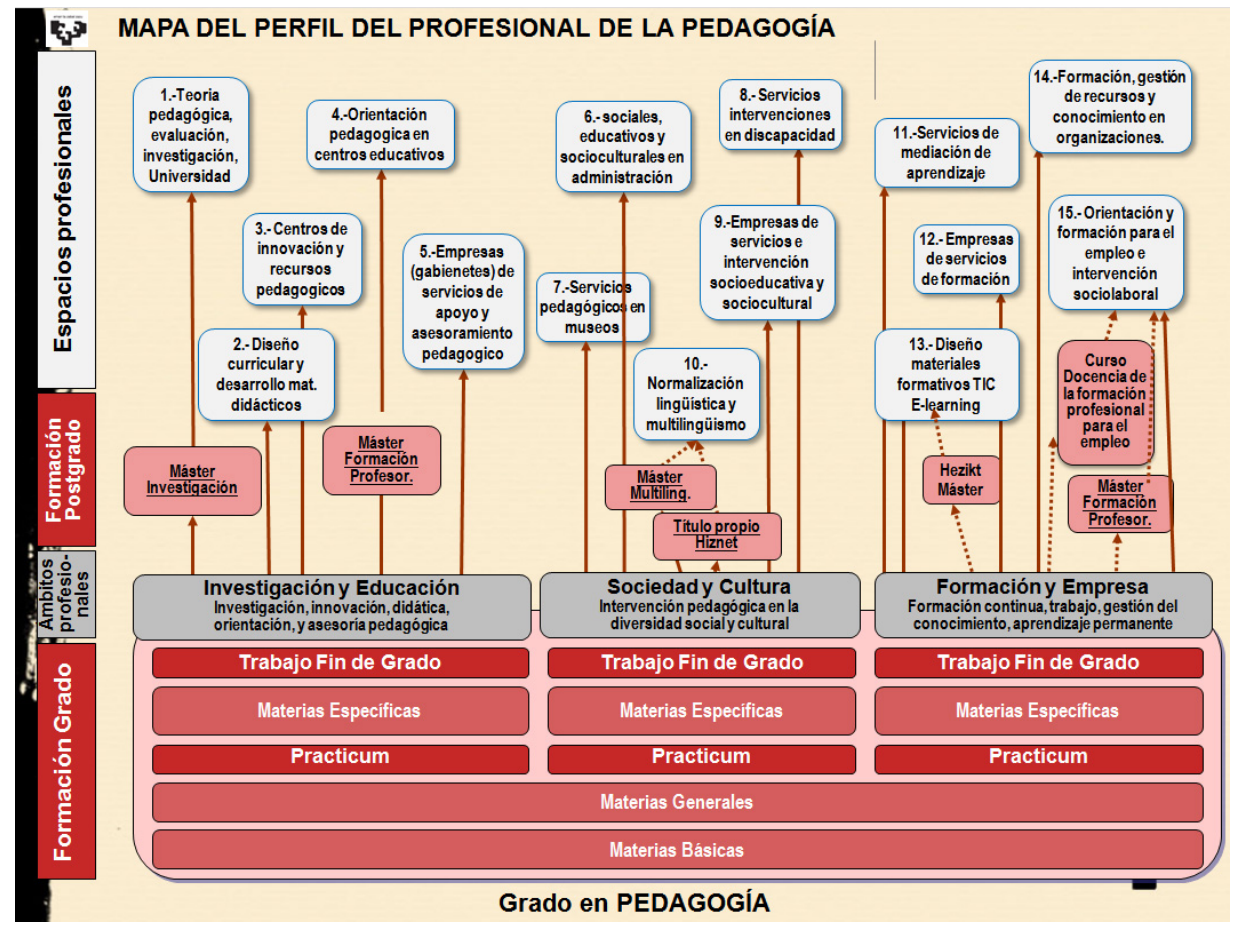

Mapa del perfil del profesional de la Pedagogía. Adaptado del mapa de profesionalización Pedagogía (https://www.ehu.eus/es/web/hefa/pedagogia) 
JON ALTUNA, ESTHER CRUZ, ANA AIERBE, ALBA MADINABEITIA E INAZIO MARKO ESTUDIO DE LOS ESPACIOS PROFESIONALES ACTUALES DE LA PEDAGOGÍA: LA VOZ DEL ALUMNADO Y DE LOS PROFESIONALES

Es este último estudio el que se tiene como referencia en esta investigación porque atiende a las variables contextuales propias del Grado de Pedagogía de la $\mathrm{UPV} / \mathrm{EHU}$.

\section{OBJETIVOS}

1. Identificar las necesidades formativas del alumnado de cara a la mejora del grado teniendo en cuenta las salidas profesionales.

2. Conocer el desarrollo de las competencias transversales en el grado de Pedagogía desde la perspectiva del alumnado.

3. Analizar la importancia que los y las profesionales de la Pedagogía conceden a las competencias transversales en función de los ámbitos de trabajo para los que se prepara el alumnado.

\section{METOdología}

El diseño utilizado en el estudio es exploratorio, descriptivo y longitudinal puesto que busca un primer conocimiento de la opinión de los agentes participantes en el fenómeno estudiado y describe con datos, porcentajes, frecuencias y medias el ámbito de estudio durante un tiempo prolongado (Dendaluce, 2002). En este caso, lo que se pretende es conocer la perspectiva del alumnado y de los profesionales de la Pedagogía en activo, por ello los y las participantes son elegidos de acuerdo con determinadas características (Kerlinger, 1983).

Se ha empleado una metodología mixta, cuantitativa y cualitativa. Es decir, tanto el alumnado como los profesionales en activo han cumplimentado un cuestionario y además se les ha realizado una entrevista (a los profesionales) y grupos de discusión (al alumnado) para poder indagar con mayor profundidad en el objeto de estudio (Dendaluce, 1999).

\subsection{Participantes}

El estudio ha contado con 283 participantes en total, de los cuales 266 son estudiantes de $4^{\circ}$ curso de Pedagogía de los cursos comprendidos entre 2014 y 2019, y 17 son pedagogos/as en activo que pertenecen a distintos ámbitos profesionales reflejados en el mapa de la titulación (ver Imagen 1).

La muestra es no probabilística y por conveniencia. Es no probabilística, no tenemos acceso a una lista completa de los individuos que forman la población puesto que el objetivo no es estimar tasas ni elaborar baremos, sino investigar las relaciones existentes entre las variables de un grupo determinado, en este caso entre las dimensiones de la encuesta de satisfacción de la titulación y las percepciones sobre la formación y las competencias de cara a las salidas profesionales del pedagogo/a. Se define por conveniencia porque los participantes se encontraban 
JON ALTUNA, ESTHER CRUZ, ANA AIERBE, ALBA MADINABEITIA E INAZIO MARKO ESTUDIO DE LOS ESPACIOS PROFESIONALES ACTUALES DE LA PEDAGOGÍA: LA VOZ DEL ALUMNADO Y DE LOS PROFESIONALES

disponibles en el momento de la aplicación (no han sido seleccionados mediante un criterio estadístico), y además pertenecen a la población de interés ya que cumplían con los criterios delimitados en la investigación. En el caso del alumnado estar en el último año del grado habiendo superado las asignaturas de los cursos previos, y en el caso de los profesionales, tener experiencia laboral en el grado y los estudios académicos afines al ámbito de estudio.

\subsection{Instrumentos de recogida y de análisis de datos}

La recogida de datos se realizó durante los cursos escolares comprendidos entre el 2014 y el 2019. Por un lado, se creó un cuestionario tipo escala Likert (1-4) para conocer la opinión y realizar un análisis de la titulación, desde la visión del alumnado de $4^{\circ}$ curso del grado de Pedagogía, en relación con los aspectos más relevantes del mismo. Las respuestas de la escala corresponden a la siguiente escala: 4 (muy buena), 3 (buena), 2 (a medias) y 1 (mala) (ver Tabla 2).

A su vez, se llevaron a cabo grupos de discusión con el alumnado de cada curso para profundizar y completar con respuestas cualitativas las recogidas en la escala.

Con relación a los profesionales se contactó con pedagogos y pedagogas de cada uno de los ámbitos recogidos en el mapa de salidas profesionales de la Pedagogía y contestaron una escala tipo Likert en función de su estimación sobre la importancia que cada asignatura del grado tiene para su labor profesional. Las respuestas se han recogido mediante la escala Likert de 0-4 donde «0" es «No tiene relevancia» y 4 es «Totalmente relevante». A su vez, se les preguntó mediante entrevistas personalizadas por las competencias necesarias para llevar a cabo su trabajo y valoraron en base a la misma escala la importancia de las competencias transversales que se trabajan en el grado de Pedagogía.

Con relación a la entrevista se tuvieron en cuenta tanto los ámbitos de trabajo del pedagogo/a como las asignaturas y los centros de prácticas del grado de Pedagogía, elaborando un guion de la entrevista semi-estructurada para los profesionales de la Pedagogía que trabajan fuera de la Universidad.

El análisis de los datos cuantitativos se realizó mediante el programa SPSS versión 25 y se realizaron las pruebas de consistencia interna de los ítems del cuestionario por categorías.

\section{Resultados}

\subsection{Identificación de aspectos para la mejora del grado}

Del análisis de las encuestas de satisfacción y la opinión del alumnado $(\mathrm{N}=266)$ de la titulación de Pedagogía (Ver Tabla 2), de cara a la identificación de aspectos que puedan mejorar el grado, se desprende que entre las dimensiones generales del grado de Pedagogía perciben que aquella que presenta el nivel más bajo es la Orientación laboral. A lo largo de los años la puntuación ha oscilado entre 1.4 y 2.0 
puntos (de un máximo de 3.7 puntos). En coherencia con los datos cuantitativos recogidos, las opiniones recabadas van en la misma dirección: "Hemos tenido este año una conferencia en la que nos contaban su experiencia ( $3^{\circ}$ curso). Es mejor si esto se biciera desde el principio, para exponer cuáles son las salidas profesionales y cuáles no, y qué formación necesitamos para ello".

Claramente, debe reforzarse la orientación académica y laboral ofertada. Para ello, algunas propuestas concretas del alumnado son: a) comenzar desde los primeros cursos $\left(2^{\circ} \mathrm{O} 3^{\circ}\right)$ con la orientación laboral sin esperar al último curso y ofrecer más información sobre las especialidades; b) impulsar más visitas de profesionales en activo para contar al alumnado su experiencia; c) procurar más salidas/visitas a entidades (empresas, diferentes entidades: «En el segundo curso, por ejemplo, hicimos una de un proyecto de innovación de un centro y yo creo que eso sirve mucho, el que se nos dé la posibilidad de ver en qué ámbitos podemos trabajar, cara a la inserción laboralı; c) utilizar recursos online y redes sociales como (Facebook, Twitter...) o Infojobs: "En Twitter abora hay un montón de gente que se dedica a becas, trabajos y tal, que está muy bien y nos podría llegar... Y por ejemplo ir visualizando eso a base de un grupo en Twitter o en Facebook»; d) trabajar la competencia de "cómo publicitarnos mejorn.

TABLA 2

Percepción del alumnado sobre las dimensiones generales del grado de Pedagogía

\begin{tabular}{|l|l|l|l|l|l|l|l|}
\hline $\begin{array}{l}\text { Indicadores (escala 1-4) / } \\
\text { Año-curso académico }\end{array}$ & 2014 & 2015 & 2016 & 2017 & 2018 & 2019 & Media \\
\hline $\begin{array}{l}\text { Organización plan de } \\
\text { Estudios }\end{array}$ & 2.6 & 2.9 & 2.4 & 2.9 & 2.5 & 2.6 & 2.6 \\
\hline Coordinación & 2.4 & 2.2 & 2.6 & 2.8 & 2.2 & 2.9 & 2.5 \\
\hline Acceso a la Información & 3.4 & 3.2 & 3.2 & 3.0 & 3.1 & 2.2 & 3.0 \\
\hline Actitud del Profesorado & 2.8 & 3.1 & 2.9 & 2.7 & 2.7 & 2.1 & 2.7 \\
\hline Instalaciones & 3.6 & 3.3 & 2.8 & 2.7 & 3.0 & 1.9 & 2.9 \\
\hline Recursos TIC & 3.1 & 2.9 & 2.6 & 2.7 & 2.7 & 2.2 & 2.7 \\
\hline $\begin{array}{l}\text { Metodología de enseñanza- } \\
\text { aprendizaje }\end{array}$ & 3.1 & 2.7 & 2.5 & 2.8 & 2.8 & 2.4 & 2.7 \\
\hline Sistemas de evaluación & 2.7 & 3.0 & 2.7 & 2.7 & 2.7 & 2.7 & 2.75 \\
\hline Resultados obtenidos & 3.2 & 2.8 & 3.2 & 2.8 & 2.8 & 2.3 & 2.9 \\
\hline Prácticas Externas & 3.4 & 2.9 & 3.0 & 2.7 & 2.7 & 2.5 & 2.9 \\
\hline Orientación laboral & $\mathbf{1 . 7}$ & $\mathbf{1 . 4}$ & $\mathbf{1 . 8}$ & $\mathbf{2 . 0}$ & $\mathbf{2 . 0}$ & $\mathbf{3 . 2}$ & $\mathbf{2 . 0}$ \\
\hline Movilidad & 2.5 & 2.9 & 2.6 & 2.3 & 2.3 & 2.7 & 2.5 \\
\hline Apoyo al aprendizaje & 3.7 & 3.5 & 2.9 & 3.0 & 3.0 & 2.1 & 3.0 \\
\hline MEDIA & $\mathbf{2 . 9}$ & $\mathbf{2 . 8}$ & $\mathbf{2 . 7}$ & $\mathbf{2 . 7}$ & $\mathbf{2 . 7}$ & $\mathbf{2 . 5}$ & $\mathbf{2 . 7}$ \\
\hline
\end{tabular}

Fuente: Elaboración propia 
JON ALTUNA, ESTHER CRUZ, ANA AIERBE, ALBA MADINABEITIA E INAZIO MARKO ESTUDIO DE LOS ESPACIOS PROFESIONALES ACTUALES DE LA PEDAGOGÍA: LA VOZ DEL ALUMNADO Y DE LOS PROFESIONALES

Respecto a la preparación al mundo laboral de las asignaturas del grado de Pedagogía (Ver tabla 3), el alumnado $(\mathrm{N}=266)$ indica que su preparación tiene que ver sobre todo con la Teoría pedagógica, evaluación e investigación (ítem 1, M=3.72) seguido de los Servicios de Intervención en Discapacidad (ítem 8, M=3.53) y, en tercer lugar, con la Orientación pedagógica en centros educativos (ítem 4, M=3.29). En el extremo opuesto, la salida profesional que menos identifican con las asignaturas del Grado es la de Servicios pedagógicos en museos (ítem 7, M=1.75), Diseño de material TIC/IKT y e-learning (ítem 13, M=2.33) así como lo relacionado con Sociedad, educación y servicios culturales (ítem 6, M=2.55). Ante esto, las propuestas de mejora que el alumnado expone en los focus group respecto a las salidas profesionales, son: conocer las funciones y salidas del pedagogo desde los primeros cursos; impulsar la autonomía de manera que el alumnado tenga margen para la toma de decisiones, organización y la planificación del tiempo; trabajar la crítica y la autocrítica; atender al liderazgo, pudiendo rotar en el rol de gestor o administrador de un grupo con cierta responsabilidad; el profesorado podría facilitar el trabajo cooperativo; trabajar la competencia digital (TICS) en más asignaturas; dar opciones más amplias para desarrollar el practicum en el contexto no formal y conocer otros ámbitos más allá de los centros educativos (empresas, entidades, gabinetes...).

TABLA 3

Percepción de la preparación al mundo laboral en el grado de Pedagogía

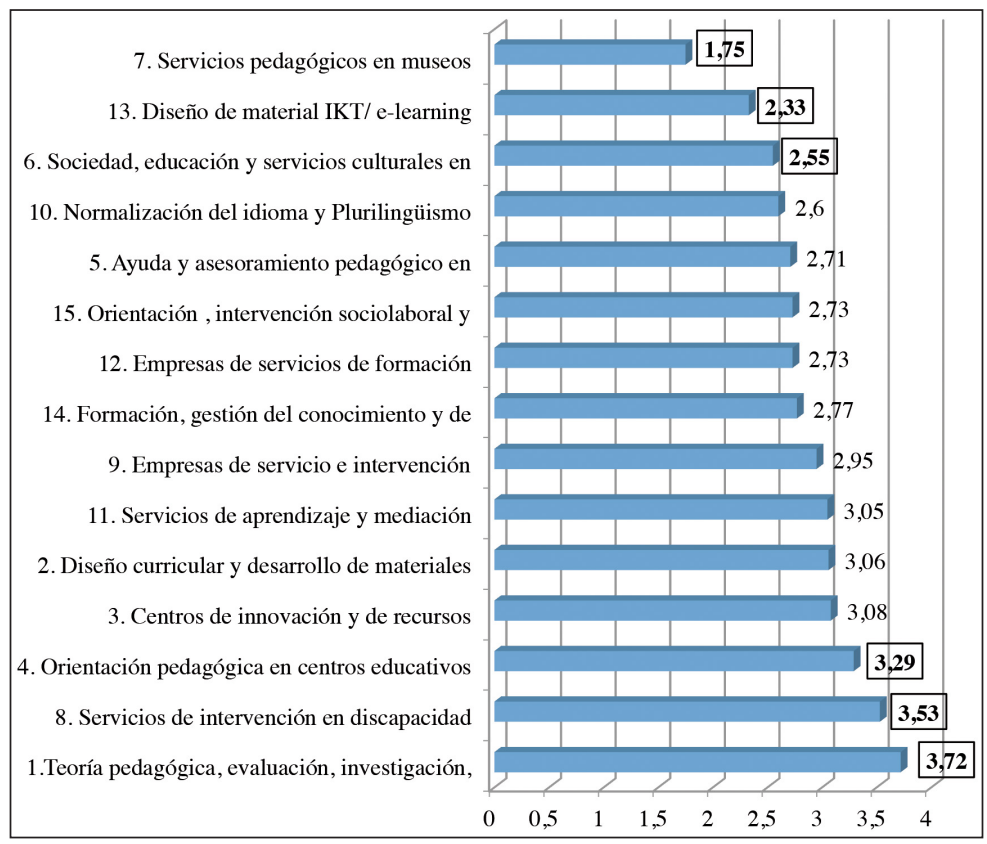

Fuente: Elaboración propia 
JON ALTUNA, ESTHER CRUZ, ANA AIERBE, ALBA MADINABEITIA E INAZIO MARKO ESTUDIO DE LOS ESPACIOS PROFESIONALES ACTUALES DE LA PEDAGOGÍA: LA VOZ DEL ALUMNADO Y DE LOS PROFESIONALES

\subsection{Las competencias transversales desde la perspectiva del alumnado}

Mediante las encuestas de satisfacción y focus group realizados con alumnado del último curso del grado de Pedagogía a lo largo de estos años se ha indagado en la identificación de las competencias transversales que, desde su perspectiva, consideran relevantes para su formación y desarrollo de la profesión. Concretamente, las preguntas para el debate e intercambio de opinión, son: a) En tu opinión, ¿Cuáles son las competencias transversales que más se han trabajado a lo largo del grado?, b) ¿Y las que menos o no se han trabajado? y, c) Ahora que estás finalizando el grado, ¿qué competencias transversales ha de llevar consigo un Pedagogo?

En general, el alumnado opina que sabe identificar las competencias transversales. A lo largo de la carrera, a excepción de algunas pocas asignaturas, dicen haberlas trabajado de un modo implícito y menos de modo explícito, aunque han sido reseñadas principalmente en las actividades interdisciplinares de módulo $\left(\mathrm{AIM}^{1}\right)$ y en los practicum. Reconocen que se centran más en las generales que en las transversales y perciben una progresión de manera que, a medida que han ido avanzando en los cursos, han adquirido mayor sensación de conocimiento de las competencias y de las funciones del pedagogo. "igual con el tiempo haciendo una reflexión, este año o el año pasado, te das cuenta de que has adquirido alguna/s en algunos temas, en el momento no te das cuenta».

Les dan importancia a todas las competencias transversales planteadas viéndolas imprescindibles para su futura inserción laboral pero, también, desde una perspectiva formativa global, para la vida, y no solo para el trabajo. Consideran que las más trabajadas son el trabajo en equipo, la comunicación oral y la comunicación escrita. En todos los trabajos del AIM se trabajan estas competencias. El trabajo en equipo está bastante extendido y trabajado, aunque señalan que con frecuencia no es un trabajo en equipo real, sino que son partes elaboradas individualmente que se plasman en un documento común. Argumentan que hacer todo entre varios ralentiza su consecución. Se alude también a la estabilidad de los grupos en el tiempo con desigual implicación de sus miembros. "Trabajo en equipo sí. Cooperativo... al final siempre vas con tu grupo. Yo be echado de menos rotar, rotar más". Reconocen dificultades a la hora del abordaje de la competencia con el conjunto de habilidades que supone: "Decimos que el trabajo en equipo está super-machacado, pero no

1. Tal y como aparece en la memoria del Grado de Pedagogía presentada a la ANECA: «El planteamiento pedagógico de la estructura modular adquiere sentido en la medida en que se materializa en una actividad global e interdisciplinar, a través de la cual el alumnado demuestra las competencias diseñadas para el módulo. Esta actividad de módulo constituye un elemento aglutinador del trabajo en equipo por parte del alumnado y del profesorado que deber coordinarse para ofertar un trabajo que supera la visión exclusivamente de su asignatura o disciplina». El Grado de Pedagogía de la UPV/EHU está constituido por 7 módulos con sendas Actividades Interdisciplinares de Módulo para cada uno de los mismos. 
hemos trabajado la otra parte del trabajo en equipo: la parte de tirar e incluso decir que la otra persona no ba becho nada".

"Hay veces que los grupos son súper estables, tú estás dando tu esfuerzo para que gente que ni le interesa ni viene a clase apruebe sus asignaturas. Y luego te perjudica a ti cuando, si lo bubieras becho solo o con la gente que de verdad le interesa trabajar, pues bubieses sacado mejores notas".

"Muchas veces somos conscientes de yo en este trabajo be hecho el $80 \%$ y esta persona el $20 \%$, pero no lo digo, y te van a poner la misma nota. Entonces, creo que tendríamos que haber sido más valientes de decir 'yo be hecho esto y esta persona, no'".

El desarrollo de la competencia oral se vincula sobre todo con las presentaciones en el aula, por lo que el alumnado propone implicarla a más contextos:

«la bemos trabajado a la hora de presentar pero luego también hay una parte de nuestro trabajo de cara a personas; adaptar tu lenguaje a la persona o situación en la que estás... 'Cómo decir, cómo transmitir'... La resolución de conflictos respecto a la profesión trabajarlos desde la perspectiva experiencial. Están ligados a la comunicación y al desarrollo de habilidades sociales (aceptar las críticas, empatía)".

El alumnado reconoce que se trabaja mucho la comunicación escrita pero que en ocasiones el seguimiento queda reducido al plano más formal (formato, normas APA) Se echa de menos la retroalimentación del profesorado: «hacer más hincapié en los conceptos y la teoría. En el practicum este año se nos pide esto, escribir de un modo técnico y científico".

Respecto a la competencia creativa y de innovación, consideran que se ha trabajado poco; no hay un acuerdo claro entre las opiniones dadas. Para algunos ha habido oportunidades para desarrollarla mientras otros creen que no las ha habido o que eso es algo propio de la persona. Perciben que muchos trabajos son excesivamente dirigidos y no ayudan a al desarrollo de esta competencia. "Se ha quedado mucho en palabras pero igual hay que ser más pragmático”.

Asimismo, perciben que la competencia de aprender a aprender se ha trabajado poco, han podido ayudar las AIM a la hora de hacer que el alumnado se vaya informando y formando sobre un tema. Reconocen que en la AIM es donde mayor número de ellas se piden en una misma tarea. "Con la AIM bemos aprendido a organizar y a construir algo propio pero no como para considerar que se realizan actividades creativas o de emprendizaje». También manifiestan que el número de estudiantes por curso y los grupos condicionan el trabajo de estas competencias. En definitiva, no se perciben actividades donde se pongan en práctica dicha competencia y sugieren: «trabajo, proyecto, problema, con esa actividad aprendo a aprender. Si me lo plantean de esa manera yo creo que se desarrolla la competencia».

\subsection{Las competencias transversales desde la perspectiva de las y los profesionales}

En este apartado se recogen las respuestas relativas a las competencias transversales de los 17 profesionales participantes. Dichos profesionales cumplimentaron 
el cuestionario y la información complementaria que 12 de ellos aportaron en las entrevistas semi-estructuradas, tanto la referida a las competencias transversales de la profesión en general (pregunta 17) como la relacionada con los ámbitos específicos (ítem 19).

Los análisis efectuados nos indican que la valoración de las distintas competencias transversales por parte de los profesionales es muy alta. En todas ellas la media de los ítems es superior a 4 dentro de una escala de 1 a 5 (ver Tabla 4). Con relación a los ámbitos de trabajo, se realizó la comparación de medias correspondiente pero no se encontraron diferencias estadísticamente significativas entre dichos ámbitos y las propias competencias.

TABLA 4

Medias de las competencias transversales

\begin{tabular}{|l|l|l|l|}
\hline & Media & D.T. & Alfa Cronbach \\
\hline Comunicación oral & 4.38 & 0.66 & .943 \\
\hline Comunicación escrita & 4.13 & 0.63 & .791 \\
\hline Competencia Comunicativa & 4.27 & 0.59 & .919 \\
\hline Aprender a aprender & 4.42 & 0.70 & .898 \\
\hline Trabajo en equipo & 4.29 & 0.80 & .960 \\
\hline Creatividad & 4.19 & 0.65 & .865 \\
\hline
\end{tabular}

Fuente: Elaboración propia

En general, del total de entrevistados-as, se han recabado 43 referencias relativas a las cinco competencias transversales (Marko et al., 2019) consideradas necesarias para el desempeño de la profesión de pedagogo-a (ver Tabla 5). Entre ellas, 35 están relacionadas con las cinco competencias transversales y otras 8 que en el cuestionario no aparecían explicitadas pero que aparecen referenciadas en otros importantes estudios como el de la red Eri-net de la UNESCO (2015): las competencias intra-personales (resiliencia, inteligencia emocional, motivación y esfuerzo) e inter-personales (empatía, escucha activa, habilidades sociales, asertividad).

Finalmente, se alude al emprendizaje y la proactividad, competencias muy relacionadas con la autonomía, la autorregulación del aprendizaje y el pensamiento crítico (Aprender a aprender). La proactividad definida como "bacer que las cosas sucedan; para ser proactiva primero tienes que tener un sueño, una inquietud, un reto; tener confianza en tu trabajo y optimismo" (Cod. 12). Asimismo, se ha indagado, en la importancia y la necesidad del trabajo de las competencias transversales que, en opinión de los y las profesionales de la Pedagogía, consideran relevantes respecto a los ámbitos profesionales específicos y se han recogido 41 referencias en total (ver Tabla 5). 
JON ALTUNA, ESTHER CRUZ, ANA AIERBE, ALBA MADINABEITIA E INAZIO MARKO ESTUDIO DE LOS ESPACIOS PROFESIONALES ACTUALES DE LA PEDAGOGÍA: LA VOZ DEL ALUMNADO Y DE LOS PROFESIONALES

TABLA 5

Competencias transversales necesarias para el desempeño de la profesión y asociadas a los diversos itinerarios profesionales o ámbitos específicos

\begin{tabular}{|l|c|c|}
\hline Competencias transversales & Profesión & Ámbitos específicos \\
\hline Aprender a aprender & $14(32.55 \%)$ & $11(26.82 \%)$ \\
\hline Comunicación & $11(25.58 \%)$ & $1(2.43 \%)$ \\
\hline Trabajo en equipo & $5(11.63 \%)$ & $19(21.95 \%)$ \\
\hline Creatividad & $5(11.63 \%)$ & $6(14.63 \%)$ \\
\hline Otras (inter- e intra- personales) & $8(18.61 \%)$ & $4(9.75 \%)$ \\
\hline
\end{tabular}

Fuente: Elaboración propia

A continuación, se exponen los resultados relativos a cada competencia transversal incluyendo los datos aportados por el cuestionario, las referencias a la profesión en general y aquellas asociadas con los ámbitos específicos. Dentro de la Competencia comunicativa (que engloba tanto la oral, escrita, no verbal y la mediática), la capacidad para la exposición estructurada de la presentación ( $M=4.53$; D.T=.71) junto con la capacidad para la utilización adecuada de medios de apoyo a la presentación (soportes, TIC...) $(\mathrm{M}=4.53$; D.T=.62) destaca la capacidad para usar fuentes diversas $(\mathrm{M}=4.41 ; \mathrm{D} . \mathrm{T}=.93)$. Sobre esta competencia comunicativa (11 referencias, $25.58 \%$ ) los profesionales entrevistados, además de aludir a la comunicación oral, no verbal y escrita, otorgan relevancia a la competencia mediática, entendida no solo como saber utilizar los recursos tecnológicos sino, también, a saber, recoger los temas con rigor, criterio de selección y pensamiento crítico. Esto está relacionado con la alfabetización mediática e informacional (UNESCO, 2015) y con la capacidad de Aprender a aprender. Al referirse a los ámbitos específicos, la única referencia apunta a «la capacidad para atraer la atención» (Cod 09) al referirse a la comunicación oral.

Dentro de la competencia Aprender a aprender la media más alta corresponde a la capacidad para mantener una actitud positiva y de motivación hacia el aprendizaje $(\mathrm{M}=4.59$; D.T $=.71)$ y la capacidad para la crítica y el compromiso ético y responsable $(\mathrm{M}=4.42 ; \mathrm{D} . \mathrm{T}=.72)$. Los profesionales, junto a la autonomía y el pensamiento crítico, destacan las siguientes destrezas (14 referencias, 32,55 \%): saber buscar la información (decretos, boletines, proyectos educativos, ayudas económicas...); saber gestionar los programas; gestionar el tiempo; aplicar pruebas estandarizadas, interpretar los datos, realizar diagnósticos y elaborar informes; conocer los itinerarios formativos, los ámbitos formal y no formal; saber planificar y organizar; saber liderar y gestionar recursos para cada colectivo. Al referirse a las competencias necesarias para los ámbitos específicos de la profesión, se alude al Aprender a aprender (11 referencias, 26.82 \%) como la competencia más importante después del trabajo en equipo, e 
incluye: la formación continua; la capacidad de análisis de la realidad; saber de dónde obtener información; el análisis de datos (recoger muchos indicadores, saber gestionarlos y sacar conclusiones para mejorar); ser metódico, tener organización en el trabajo; y/o aprender mediante gamificación de juegos.

Otra de las competencias transversales consideradas necesarias para la profesión del pedagogo es el Trabajo en equipo, en especial la capacidad para la planificación del equipo $(\mathrm{M}=4.35 ; \mathrm{D} . \mathrm{T}=.78)$ y la capacidad para la resolución de conflictos y problemas colaborativos ( $\mathrm{M}=4.35$; $\mathrm{D} . \mathrm{T}=.86)$. Concretamente aluden a ( 5 referencias, $11,66 \%$ ): saber crear y dinamizar grupos; desempeñar diferentes roles y perfiles; y enseñar a educadores-as cómo mediante actividades lúdicas puede darse el desarrollo emocional, cognitivo y social, entre otras. Llama la atención, que cuando hacen referencia a las competencias transversales asociadas a los diversos itinerarios profesionales, los entrevistados sitúan en primer lugar de importancia el trabajo en equipo (19 referencias, $21.95 \%$ ) que, en su opinión, implica: saber compartir el conocimiento (y/o la información) y el aprendizaje; retroalimentación; saber situarse ante conflictos y gestionarlos; trabajar en red y compartir; y actitud abierta en el sentido de "tener la capacidad de abrirte a la opinión de otro/a para ver que no todo es como lo tenías programado, que pueden surgir imprevistos" (Cod. 06).

Respecto a la Creatividad, destaca que la media más alta se posiciona en la capacidad para adaptarse a las situaciones cambiantes, modificando la conducta para integrarse, con versatilidad y flexibilidad (M=4.59; D.T=.61). La opinión de los profesionales sobre esta competencia refuerza y complementa lo anteriormente señalado, ya que destacan (mediante 5 referencias, 11,63 \%): la capacidad para plantearse nuevos retos, en el sentido de sproponer actividades rompedoras que te obligan a estar en situaciones nuevas" (Cod. 02) y la flexibilidad, versatilidad o adaptación a los cambios, tal y como expresa esta entrevistada: «flexibilidad y versatilidad, algo que necesariamente aparece entre las funciones del puesto de trabajo. Pero, también, es necesario estar preparada y ser capaz de realizar otro tipo de requerimientos para trabajar con distintos grupos, para profundizar en temas nuevos. No tiene nada que ver lo que hacíamos hace 15 años con lo de hoy en día. Hay que adaptarse» (Cod. 07). Esto apunta en la misma dirección que la dimensión competencial que la red Eri-net, (UNESCO, 2015) denomina Pensamiento crítico e innovador.

Por otra parte, la media más baja corresponde $(\mathrm{M}=3.88 ; \mathrm{D} . \mathrm{T}=.85)$ a la Orientación a la calidad, utilizando indicadores de evaluación, y buscando la mejora continua. Al referirse a los ámbitos específicos de la Pedagogía, también, la Creatividad se menciona ( 6 referencias, $14.63 \%$ ) bien en un sentido general como adaptación al cambio, es decir, la "capacidad para hacer frente a las nuevas situaciones" (Cod. 09) o bien en referencia al diseño y adaptación de materiales como, por ejemplo, "el diseño y adaptación de juegos» (Cod. 11).

Finalmente, cuando se les pregunta por las competencias transversales, los entrevistados mencionan, por primera vez, competencias de carácter sistémico: entre otras, proponer a las entidades nuevos temas y/o proporcionarles materiales-recursos 
JON ALTUNA, ESTHER CRUZ, ANA AIERBE, ALBA MADINABEITIA E INAZIO MARKO ESTUDIO DE LOS ESPACIOS PROFESIONALES ACTUALES DE LA PEDAGOGÍA LA VOZ DEL ALUMNADO Y DE LOS PROFESIONALES

nuevos. No obstante, en la pregunta dirigida a las competencias transversales de la profesión en general (ítem 17), cuando se les pregunta por aquellas relacionadas con los ámbitos específicos (ítem 19: saber situarse en la organización y/o sentirse parte de un proyecto compartido dentro de la organización) como, por ejemplo, "creer en un trabajo en conjunto. En las organizaciones sociales esto debe ser importante, sentirse parte del proyecto" (Cod. 06), estas competencias sistémicas no son mencionadas.

\section{CONCLUSIONES}

La voz del alumnado y de los profesionales es fundamental para la mejora de los grados y, por ende, de las universidades.

Identificar las necesidades formativas del alumnado con relación a las competencias transversales de la titulación de cara a la inserción laboral del mismo, era una necesidad clave para la mejora continua del Grado de Pedagogía. Entre las dimensiones generales del grado de Pedagogía, según la percepción del alumnado en el transcurso de los años 2014-2019, aquella que presentaba el nivel más bajo era la Orientación laboral y por eso era necesario este estudio. Debíamos incorporar en el grado de Pedagogía, acciones que supusieran un puente entre los estudios de grado, poniendo sobre la mesa todas las asignaturas de este, y la profesión o salidas profesionales; y ese puente no podía ser otro que las competencias transversales implicadas en ambos contextos.

Hay algunas asignaturas que tienen más relación con las salidas profesionales que otras, así pues, destacamos principalmente asignaturas que se imparten en tercer y cuarto curso y que son las que tienen un carácter de especialización. Es precisamente en estos cursos donde se sitúan las 5 asignaturas con más puntuación por parte del alumnado.

Hay salidas profesionales, partiendo del mapa profesional de la Pedagogía, que están más representadas que otras según el alumnado como son en servicios de intervención en discapacidad, orientación pedagógica en centros educativos y sobre todo teoría pedagógica, evaluación e investigación.

Los profesionales identifican algunos aspectos a mejorar con relación a las competencias transversales en el grado de Pedagogía aunque todos valoran como fundamentales las 5 competencias transversales definidas para Pedagogía.

Estos profesionales piensan que además de estas competencias transversales, se debería impulsar más el trabajo de otras como la empatía, la adaptación al cambio, la capacidad para la atraer la atención, que, si bien no están explícitamente planteadas, sí que las podemos considerar incluidas dentro de las 5 competencias definidas como transversales para Pedagogía.

Respecto a la percepción del alumnado de las competencias transversales, segundo objetivo de este trabajo, los resultados coinciden con los hallados por la investigación previa de Eizagirre et al. (2017) y, en cierto modo, los complementan. 
En efecto, en opinión del alumnado, las competencias transversales más trabajadas son el trabajo en equipo, la comunicación oral y escrita. Respecto al trabajo en equipo, siendo una de las competencias priorizada por los profesionales, según el alumnado no se trabaja de manera adecuada ya que, según los mismos, no hay trabajo colaborativo.

Se constata la conveniencia de ofrecer mayor explicitación y clarificación de cada una de las competencias por parte del alumnado; la necesidad de "feedback» del desarrollo de cada competencia por parte del profesorado y trabajarlas más desde una perspectiva personal. No obstante, incluso en las que refieren que son más trabajadas, debe ahondarse más en la "cualidad" de las mismas como, por ejemplo, lo relativo a la diferenciación entre el mero trabajo en grupo y el trabajo colaborativo. Como Marko et al. (2019) sugieren, un aspecto esencial es impulsar la reflexión en el alumnado induciendo una mayor implicación personal en este proceso.

Respecto al tercer objetivo planteado, analizar la importancia de las competencias transversales para el desempeño de la profesión y, en particular, en función de los ámbitos profesionales para los que se preparan los estudiantes, a modo de síntesis, Aprender a Aprender y Comunicación son las competencias transversales más citadas en relación a la profesión en general, mientras en las específicas del ámbito concreto de la Pedagogía al que se dedican, la competencia de Aprender a Aprender pasa a un segundo lugar priorizando la del trabajo en equipo, a la vez que se da un paso hacia una visión más sistémica (por ej. sentirse parte de un proyecto compartido dentro de la organización) refiriéndose a las competencias de Ciudadanía global (red Eri-net, UNESCO). Ahora bien, dado que los profesionales dentro de la competencia creativa la puntuación media más baja la han otorgado a la orientación a la calidad, utilizando indicadores de evaluación, y buscando la mejora continua, este es un aspecto al que se le debe prestar especial atención en futuros estudios.

La trascendencia del estudio radica en su carácter longitudinal, de mejora continua del Grado tratando de integrar las voces tanto de profesionales como del alumnado, complementada y ampliada por herramientas diversas (cuestionarios de satisfacción al alumnado, cuestionarios a profesionales, entrevistas, grupos de discusión). En este sentido, complementa y supone un avance en la investigación en este campo, en la línea de otros trabajos (Rodríguez-Martín, 2017; Salinas, Morales y Martínez, 2008; Calvo y Susinos, 2010; Surdez, Sandoval y Lamoyi, 2018). Si bien el estudio se sitúa en una institución concreta, y por lo tanto, los resultados no son generalizables, apunta hacia aspectos relevantes para hacer confluir formación y desempeño profesional. No obstante, una línea futura de investigación es aquella cuya finalidad sea ahondar en las competencias "clave» para cada uno de los tres ámbitos y de los espacios específicos de la Pedagogía. 
JON ALTUNA, ESTHER CRUZ, ANA AIERBE, ALBA MADINABEITIA E INAZIO MARKO ESTUDIO DE LOS ESPACIOS PROFESIONALES ACTUALES DE LA PEDAGOGÍA: LA VOZ DEL ALUMNADO Y DE LOS PROFESIONALES

\section{REFERENCIAS BIBLIOGRÁFICAS}

Calvo, A. y Susinos, T. (2010). Prácticas de Investigación que escuchas la voz del alumnado: Mejorar la universidad indagando la experiencia. Profesorado. Revista de Currículum y formación del Profesorado, 14(3), 75-88. https://recyt.fecyt.es/index.php/profesorado/ article/view/42892

Dendaluce, I. (1999). La investigación educativa ante el tercer milenio. Bordón, 51(4), 363-376.

Dendaluce, I. (2002). Ciencias sociales e investigación: diversidad, debates y soluciones. En Ciencia y cultura vasca, y redes telemáticas, Actas del XV Congreso de Estudios Vascos. http://www.eusko-ikaskuntza.eus/es/publicaciones/ciencias-sociales-e-investigacion/ art-8177/

Eizagirre, A., y Altuna, J. (2014). Análisis del desarrollo de las Competencias Transversales para la empleabilidad en centros de Formación Profesional de la Comunidad Autónoma del País Vasco. Procedia-Social and Behavioral Sciences, 139, 314-320. https://doi. org/10.1016/j.sbspro.2014.08.005

Eizagirre, A., Altuna, J., y Fernández, I. (2017). Prácticas de éxito en el desarrollo de competencias transversales en centros de Formación Profesional del País Vasco. Revista española de pedagogía, 267, 293-308. https://doi.org/10.22550/REP75-2-2017-7

Eizagirre, A., Altuna, J., Pikabea, I., Marko, J. I., y Pérez, V. (2017). Las competencias transversales en el grado de Pedagogía: diagnóstico y estado de la cuestión. REDU. Revista de Docencia Universitaria, 15(1), 259-276. https://doi.org/10.4995/redu.2017.6044

Hargreaves, A. (2003). Enseñar en la sociedad del conocimiento. Octaedro.

Kerlinger, F. (1983). Investigación del Comportamiento. Técnicas y Metodología. (2. ${ }^{a}$ ed). Interamericana,

MacFarlane, B. (2008). El servicio en la vida académica. En R. Barnett (Ed.), Para una transformación de la universidad. Nuevas relaciones entre investigación, saber y docencia (pp. 215-230). Octaedro.

Marko, J. I., Pikabea, J. I., Altuna, J., Eizagirre, A., y Perez-Sostoa, M. V. (2019). Propuesta para el desarrollo de competencias transversales en el Grado de Pedagogía. Un estudio de caso. Revista Complutense de Educación, 30(2), 381- 398. https://doi.org/10.5209/ RCED. 57490

Prades, A. (2006). Les competències transversals i la formació universitària. (Memoria para optar al título de Doctor). Universitat de Barcelona, Barcelona, España. http://hdl. handle.net/10803/2346

Real Decreto 1393/2007, de 29 de octubre, por el que se establece la ordenación de las enseñanzas universitarias oficiales. (2007). Boletín Oficial del Estado, 260, de 30 de octubre de 2007. https://www.boe.es/eli/es/rd/2007/10/29/1393/con

Rekalde, I., y Bujan, K. (2014). La e-Rúbricas ante la evaluación de las competencias transversales en la Educación Superior. Revista Complutense de Educación, 25(2), 355-74. https://doi.org/10.5209/rev_RCED.2014.v25.n2.41594

Rodríguez-Martín, A. (2017). Prácticas innovadoras inclusivas. Retos y oportunidades. Servicio de Publicaciones de la Universidad de Oviedo.

Salinas, A. Morales, J. A., y Martínez, P. (2008). Satisfacción del estudiante y calidad universitaria: un análisis explicatorio en la unidad académica multidisciplinaria agronomía y 
JON ALTUNA, ESTHER CRUZ, ANA AIERBE, ALBA MADINABEITIA E INAZIO MARKO ESTUDIO DE LOS ESPACIOS PROFESIONALES ACTUALES DE LA PEDAGOGÍA: LA VOZ DEL ALUMNADO Y DE LOS PROFESIONALES

ciencias de la Universidad Autónoma de Tamaulipas, México. Revista de Enseñanza Universitaria, 31, 39-55. http://institucional.us.es/revistas/universitaria/31/4SalinasGuti.pdf Surdez, E. G., Sandoval, M del C., y Lamoyi, C. L. (2018). Satisfacción estudiantil en la valoración de la calidad educativa universitaria. Educación y Educadores, 21(1), 9-26. https:// doi.org/10.5294/edu.2018.21.1.1

UNESCO. (2015). Asia-Pacific Education Research Institutes Network (ERI-Net) Regional Study on Transversal Competencies in Education Policy \& Practice (Phase 1). Regional Synthesis Report. https://unesdoc.unesco.org/ark:/48223/pf0000231907

UNESCO. (2016). Preparing and Supporting Teachers in the Asia-Pacific to Meet the Challenges of Twenty-first Century Learning. https://unesdoc.unesco.org/ark:/48223/pf0000246852

Uranga, M.J., Cruz, E., Eizagirre, A.I., Gil, P., Losada, D., y Ruíz de Gauna, P. (2019). Catálogo de competencias transversales de la UPV/EHU. Servicio Editorial de la Universidad del País Vasco.

https://www.ehu.eus/documents/1432750/12757375/Cat\%C3\%A1logo+de+Competencias+tr asnversales_cas.pdf/5dd00732-9e32-5e52-0ee2-2128d9a6867b

Villa, A. (2004). Libro blanco: Título de grado en Pedagogía y educación social. ANECA. http:// www.aneca.es/var/media/150392/libroblanco_pedagogia1_0305.pdf http://www.aneca. es/var/media/150396/libroboanco_pedagogial2_0305.pdf 